\title{
FINAL REPORT \\ U.S. Department of Energy \\ DEVELOPMENT OF AN IN-SITU MICROSENSOR FOR THE MEASUREMENTS OF CHROMIUM AND URANIUM IN GROUNDWATER AT DOE SITES
}

Project Number:DE-FGO7-96ER62306

PI: Joseph Wang

Institution: NMSU

Period: Oct. 1996-Sept. 2000 


\section{Table of Contents}

2. Table of Contents

3. Executive Summary

4. Research Objectives

5. Methods and Results

6. Relevance and Impact

7. Productivity

8. Personnel

9. Publications

10. Interactions

11. Literature Cited

12. Feedback

12

13. Appendix

12 


\section{Executive Summary}

The goal of this program is to develop and test miniaturized analyzers for field monitoring trace levels of chromium $(\mathrm{Cr})$ and uranium(U). Two avenues, based on the highly sensitive electrochemical stripping analysis, have been explored for achieving the goal of field monitoring of $\mathrm{Cr}$ and $\mathrm{U}$. This technique is based on an adsorptive accumulation of the metal complex, followed by electrochemical measurement of the surface complex. A novel hand-held micromachined hand-held flow stripping analyzer and remotely deployed submersible stripping probes have thus been developed and characterized. Both routes should allow testing of these and other trace metals more rapidly and easily in a field setting. Hence, these new devices should lead to a substantially more effective and economic monitoring of priority metal pollutants and should thus have an enormous impact upon pollution control and prevention in DOE sites. 


\section{Research Objectives}

The goal of this program is to develop, optimize and deploy a siliconbased micromachined stripping analyzer for field monitoring trace levels of chromium and uranium. Such system will integrate the sample-handling steps and necessary chemical reactions (using a flow-injection operation) with the actual adsorptive stripping measurement on a small planar chip. Besides the drastic reduction in the size of the analytical system, such miniaturization should lead to increased speed, minimal reagent consumption and disposal, higher sensitivity and improved precision, and would thus revolutionize the way by which toxic metals are being monitored.

\section{Methods and Results}

This report summarizes our activity over the 4 years of the project (10/96-9/00). In accordance to our original goals, this effort has led to the replacement of conventional, lab-based, electrochemical stripping protocols and systems with new innovative strategies for field monitoring of trace chromium and uranium, based on micromachined hand-held total stripping analyzers, in-situ sensing devices and submersible microsystems. This DOEsponsored activity has resulted in 16 research papers (in major journals), two patents (see list below) and twelve presentations (including several plenary lectures in international meetings). The newly developed miniaturized instruments and microsystems played a key role in selecting the PI as the recipient of the 1999 ACS Award for Analytical Instrumentation.

The need for on-site and continuous monitoring of trace metals in a variety of environmental matrices has led to the adaptation of electrochemical stripping analysis for these tasks. Stripping analysis has been established as a powerful technique for determining toxic metals in environmental samples. The remarkable sensitivity of stripping analysis is attributed to its unique 'built-in' preconcentration (electrodeposition) step. The portable instrumentation and low power demands of stripping analysis satisfy many of the requirements for field measurements of trace metals. Our initial effort, during the FY1997 period, was aimed at improving the stripping procedures for $\mathrm{U}$ and $\mathrm{Cr}$. Traditionally, such protocols have relied on the adsorptive accumulation of $\mathrm{Cr}$ and $\mathrm{U}$ complexes with DTPA and propyl gallate (PG), respectively. To facilitate the field monitoring, we 
developed new adsorptive stripping procedures that allow the simultaneous and rapid detection of trace (ppb) $\mathrm{Cr}$ and $\mathrm{U}$. One scheme for such simultaneous detection of these target metals involves the use of a mixed ligand (DTPA/PG) solution and appearance of well-resolved and defined $\mathrm{Cr}$ and U peaks. (1). Alternately, we employed a single (but more 'universal') ligand - cupferron - for the simultaneous monitoring of $\mathrm{Cr}$ and $\mathrm{U}$ (2). Experimental parameters for both protocols were optimized systematically to assure that such simultaneous measurements do no compromise the attractive performance of single-ligand/single-metal protocols. Applicability to groundwater samples was demonstrated. While stripping measurements of $\mathrm{Cr}$ and $\mathrm{U}$ have traditionally relied on the use of mercury drop electrodes, such electrodes are not compatible with micromachining systems. Accordingly, we demonstrated the utility of iridium microelectrodes for such trace adsorptive stripping analysis of these target metals (3), optimized new $\mathrm{Ct}$ and $\mathrm{U}$ protocols, and demonstrated their attractive performance.

Two avenues have been explored for achieving the goal of field monitoring of $\mathrm{Cr}$ and $\mathrm{U}$, as well as of other relevant toxic metals. These included a micromachined hand-held flow stripping analyzer and a remotely deployed submersible stripping probes. Both routes should allow testing of trace metals more rapidly and easily in a field setting.

According to our original goals, we have designed and fabricated different configurations of the micromachined metal analyzer (e.g., Figure 1). Such hand-held flow microsystems integrate the sample processing step with the stripping detector (4). This includes coupling all the necessary steps of the adsorptive stripping assay (including the sample and ligand transport, complex formation, adsorptive accumulation, and voltammetric measurement). We used an arrangement of functional modules $(24 \times 24 \mathrm{~mm})$, stacked on top of each other, to form the miniaturized flow system (Figure 1). These modules include the sample and ligand reservoirs, micropumps, mixer, reaction coil, flow detector, and electronic control. This microsystem thus represents the smallest metal analyzer reported to date. Connecting holes allow the fluid flow. The detector has a flow-through channel with an array of iridium microelectrodes, along with reference and counter electrodes, prepared by a photolithographic process. Such dramatic scaling down has been challenging (from the microfabrication angle) and required proper attention to various obstacles (e.g., solution leaks, pump stability, peeling off of electrode layers). Overcoming these difficulties led to a very attractive performance. The high-quality response is attributed to the powerful on-chip microprocessor-controlled potentiostat $(24 \times 60 \mathrm{~mm})$, 
controlling the stripping detection process (including its baseline fitting and noise filtration). Various experimental parameters, such as flow rate and ligand concentration, were optimized for optimal performance. These studies have demonstrated also the ability to reproducibly move and mix reagents prior to the actual stripping detection step. Other versions/configurations of the micromachined stripping analyzer were designed and tested. Such micromachined stripping analyzers thus allow testing for trace metals to be performed more reliably, easily and inexpensively in a field setting. In a related 'Lab-on-a-Chip' study, we developed we developed a new microsystem, integrating a micromachined separation (electrophoresis) chip with a thick-film electrochemical detector (5). The new system obviates the need for permanent attachment of the detector, to allow rapid replacement of the electrode (as needed, e.g., in cases of surface modification or fouling).

Remotely deployable submersible sensors, capable of monitoring metal contaminants both in time and location, are advantageous for environmental monitoring. Parallel to the development of hand-held microsystems, we designed new in-situ probes for the target $\mathrm{Cr}$ and $\mathrm{U}$ metals

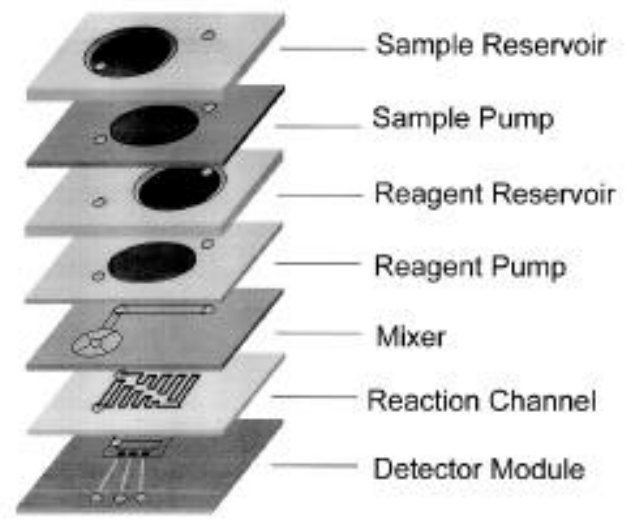

Figure 1. View of the stacked micromachined flow analyzer for adsorptive stripping monitoring of metal contaminants. (From ref. 5)

(6-8). The adaptation of the adsorptive stripping protocols of $\mathrm{Cr}$ and $\mathrm{U}$ for such in-situ monitoring required an advanced remote probe design capable for performing the complexation reaction on cable platform. Accordingly, we developed (and patented) a novel renewable-reagent remote probe (Figure 2) integrating internal ligand delivery, its complexation reaction with the metal collected in a semi-permeable microdialysis sampling tube, and 
transport of the complex to the downstream detector $(6,7)$. Our initial chromium probe was designed for delivering internally the ligand and catalyst (DTPA- $\mathrm{NO}_{3}{ }^{-}$) essential for adsorptive-catalytic measurements of trace $\mathrm{Cr}$. The resulting in-situ sensor offered a very attractive performance, with a remarkably low detection limit of $100 \mathrm{ppt} \mathrm{Cr}$, good precision and stability (e.g., RSD of $6 \%$ at $25 \mathrm{ppb}$ ), with no carry-over (memory) effects (e.g., Fig. 3), and $\mathrm{Cr}(\mathrm{III}) / \mathrm{Cr}(\mathrm{VI})$ speciation/discrimination capability. A stable response was observed for both $\mathrm{Cr}$ (VI) and $\mathrm{Cr}$ (III) species. Similar performance characteristics were demonstrated for remote monitoring of trace uranium, in connection to the internal delivery of propyl gallate (8).

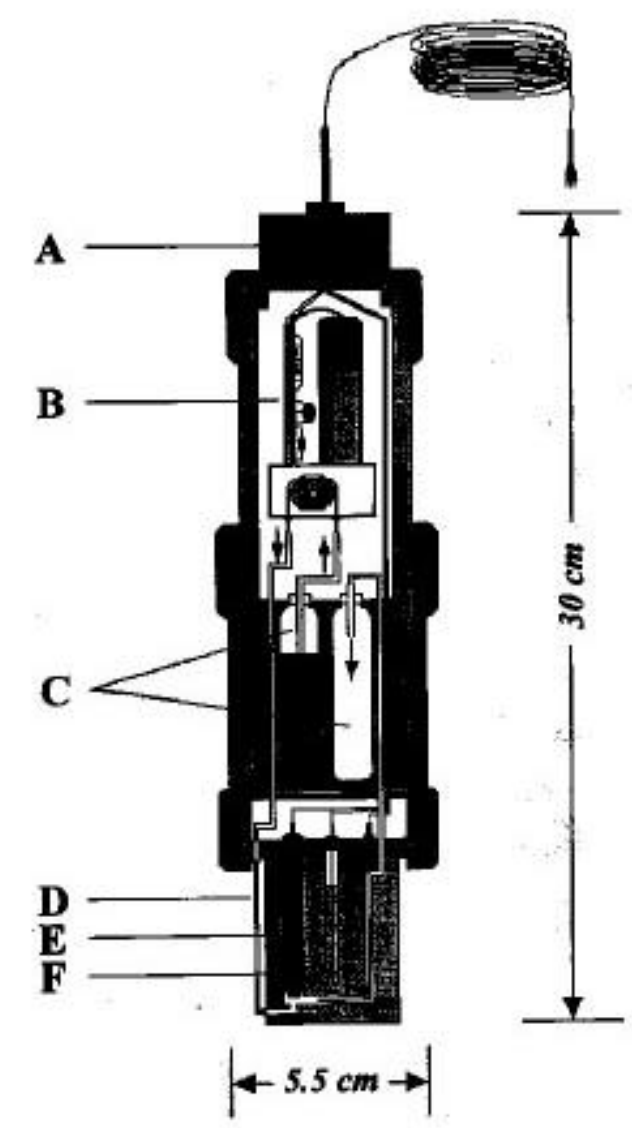

Figure 2. Renewable-reagent remote probe for in-situ monitoring of $\mathrm{Cr}$ and $\mathrm{U}$, based on internal ligand delivery, microdialysis sampling, and electrochemical detection of the complex. 


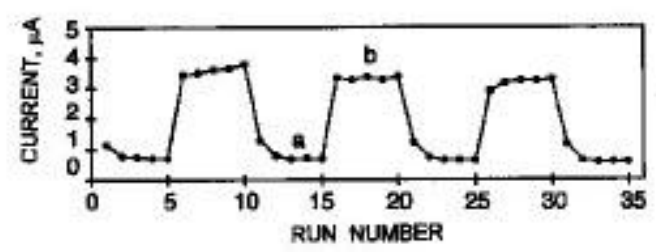

Figure 3. Performance of the in-situ probe for monitoring trace Cr: (A) Stability and (B) carry-over experiments. (A) Repetitive measurements of $25 \mathrm{ppb} \mathrm{Cr}(\mathrm{VI})$; response to alternate exposures to low (5ppb) and high (25ppb) $\mathrm{Cr}(\mathrm{VI})$ levels. Preconcentration time, 30 sec. (From ref. 6.)

Performing various sample manipulations on the cable platform was shown to offer additional advantages. For example, a 'built-in' acidification capability was employed for monitoring the total metal concentration (9). Such on-cable acidification of the collected samples offered an in-situ release (decomplexation) of metals bound to various ligands. This was documented for samples rich with NTA and EDTA. The versatility of the new submersible 'microlaboratory' was demonstrated also for circumventing in-situ common problems associated with stripping metal analysis (10). In particular a judicious choice of the internal solution was shown useful for addressing the formation of intermetallic compounds or overlapping signals. These were accomplished through an internal delivery of a third element or a masking agent, respectively. Additional efforts in the direction of remote electrochemical sensors have led to the developments of useful probes for in-situ monitoring of trace mercury (11) and nitroaromatic explosives, such as TNT (12), in connection to potentiometric stripping and square-wave voltammetric detection, respectively. We were also invited by the Italian Government for testing our remote probe for monitoring in situ the fate and gradient of toxic metals in the canals and lagoons of Venice (13).

In another effort we demonstrated that bismuth-coated electrodes offer an extremely attractive stripping performance, that compares favorably with that of mercury electrodes (14). Such bismuth film electrodes are prepared in-situ, by adding $0.4 \mathrm{ppm}$ bismuth(III) directly to the sample solution, and simultaneously depositing the target heavy metals and bismuth. Bismuth is an environmentally-friendly element, with very low toxicity, and a 
widespread pharmaceutical use. The new bismuth electrodes offer a welldefined, undistorted and highly reproducible response, high hydrogen evolution, with favorable signal-to-background characteristics comparable to those of common mercury electrodes. The new bismuth electrode offers also excellent resolution and reproducibility, and other attractive performance characteristics, which compare favorably with that of common mercury films.

\section{$\underline{6 .}$ Relevance and Impact}

The new electrochemical devices should have an enormous impact upon pollution control and prevention, as they should lead to a substantially more effective and economic monitoring of priority metal pollutants.

The project combined fundamental and practical aspects of electroanalysis and microsystems to meet the monitoring and sensing needs of DOE sites. It generated tremendous interest by private industries and other government and state agencies.

\section{Productivity}

This has been an extremely productive project. The various developments have been summarized in 18 publications in major international journals.

\section{Personnel}

Dr. J. Wang - PI

Dr. B. Tian - Res. Assoc.

J. Lu - Grad. student

J. Wang - Res. Asst.

D. MacDonald - Undergrad. student

\section{Publications}

1. "Renewable Reagent Electrochemcial Sensor for Monitoring Trace Metals", J. Wang et al., Anal. Chem. 69(1997)2640. 
2. "Simultaneous Adsorptive Stripping Voltammetric Measurements of Trace Chromium, Uranium, and Iron", J. Wang et al., Electroanalysis 9(1997)1247.

3. "Flow Probe for In-Situ Electrochemical Monitoring of Trace Chromium", J. Wang, J. Lu, and K. Olsen, , Analyst, 124(1999)349.

4. "Simultaneous Measurements of Trace $\mathrm{Cr}$ and U using Mixed Ligand Stripping Analysis", J. Wang, J. Wang, J. Lu, D. Luo, and B. Tian, Anal. Chim. Acta 354(1997)275.

5. "Remote Electrochemical Sensors", J. Wang, Trends Anal. Chem. 16(1997)84.

6. "Remote Electrochemical Sensor for Monitoring Trace Mercury", J. Wang et al., Electroanalysis 10(1998)399.

7. "Electrochemical Flow Sensor for in-situ Monitoring of Total Metal Concentrations", J. Wang et al., Anal. Communications, 35(1998)241.

8. "Adsorptive Stripping Measurements J. Wang et al., Anal. Chem., 69(1997)1657.

9. "Stripping Analysis into the $21^{\text {st }}$ Century: Faster, Smaller, Cheaper and Better”, J. Wang,, B. Tian, J. Lu, C. Olsen and C. Yarnitsky, Anal. Chim. Acta, 385(1999)429.

10. "In-Situ Flow Probe for Improving the Performance of Electrochemical Stripping Analysis", J. Wang,J. Lu, D. MacDonald and M. Augeli, (invited paper), Fres. Z. Anal. Chem., 364(1999)28.

11. "Hot-Wire Stripping Potentiometric Measurments of Trace Mercury", J. Wang, P. Grundler, M. Jasinski, and B. Tian, Anal. Chim. Acta, 396(1999)33.

12. "Renewable Reagent Enzyme Inhibition Biosensor for Remote Monitoring of Cyanide", J. Wang, B. Tian, J. Lu and D. Luo, Electroanalysis, 10(1998)1034.

13. "Anodic Stripping with Collection at Interdigitated Carbon Film Microelectrode Arrays", J. Wang, D. Lou and T. Horiuchi, Electroanalysis, 10(1998)107.

12. "Adsorptive Stripping Analysis of Trace Nickel at Iridium-based Ultramicroelectrode Arrays", J. Wang, W. Adeniyi, and S. Kounaves, Electroanalysis , 12(2000)44.

13."Remote Stripping Sensor for Determining Copper in the Lagoon of Venice", S. Daniele, C. Bragato, J. Wang, and J. Lu, Analyst, 125(2000)731.

14. J. Wang, "From Remote Sensors to Submersible labs", (invited paper), Lab. Robotics and Automation, 12(2000)178. 
15. "Lab-on-a-Cable for Monitoring Priority Contaminants", J. Wang, J. Lu, S. Ly, M. Vuki, W. Adeniyi, R. Armenderiz, Anal. Chem., 71 (2000)2659.

16. "Bismuth-Coated Carbon Electrodes for Anodic Stripping Voltammetry", J. Wang, J. Lu,S. Hocevar, P. Farias and B. Ogorevc, Anal. Chem., $72(2000) 3218$.

\section{Interactions}

The results were presented in twelve presentations in major national and international meetings and DOE workshops, including several plenary lectures in international meetings. It involved extensive collaboration with K. Olsen of our PNL sub-contract.

\section{Transitions}

The new knowledge and technology is being tested at the Hanford Site. On-going commercialization efforts, (by INW Inc.) coupled with regulatory acceptance, should lead to the translation of these research efforts into large-scale environmental applications.

\section{Patents}

1. J. Wang and K. Olsen, "Remote Electrochemical Sensor", U.S. Patent No. 5,676, 820 (1997).

2. J. Wang and K. Olsen, "Renewable Electrochemical Sensors", U.S. Patent No. 5,942, 103 (1999).

\section{Future Work}

On-going efforts are aimed to further improve the technology and to expand it towards additional metal contaminants.

\section{4. $\quad$ Literature Cited}

1. J. Wang, J. Lu, D. Luo, and B. Tian, Anal. Chim. Acta 354(1997)275.

2. J. Wang, J. Lu, and B. Tian, Electroanalysis 9(1997)1247.

3. J. Wang, B. Tian, and M. Jian, Anal. Chem., 69(1997)1657.

4. J. Wang, B. Tian, J. Lu, C. Olsen and C. Yarnitsky, Anal. Chim. Acta, $385(1999) 429$.

5. J. Wang, B. Tian, E. Sahlin, Anal Chem., 71(1999) 5436. 
6. J. Wang, J. Lu, D. MacDonald, and K. Olsen, Analyst, 124(1999)349.

7. J. Wang and K. Olsen, "Renewable Electrochemical Sensors", U.S. Patent No. 5,942, 103 (1999).

8. J. Wang, J. Lu, and K. Olsen, Anal. Chem. 69(1997)2640.

9. J. Wang, B. Tian, and J. Wang, Anal. Communications, 35(1998)241.

10. J. Wang,J. Lu, D. MacDonald and M. Augeli, (invited paper), Fres. Z. Anal. Chem., 364(1999)28.

11. J. Wang, B. Tian, and D. MacDonald, Electroanalysis 10(1998)399.

12. J. Wang, R. Bhada, J. Lu and D. MacDonald, Anal. Chim. Acta 361(1998)85.

13. S. Daniele, C. Bragato, J. Wang, and J. Lu, Analyst,125(2000)731.

14. "Bismuth-Coated Carbon Electrodes for Anodic Stripping Voltammetry", J. Wang, J. Lu,S. Hocevar, P. Farias and B. Ogorevc, Anal. Chem., 72(2000)3218.

15. Feedback: None

16. Appendix: None 